\title{
Stimulation Actuator for Walking Support: Two-Point Threshold on Planta Pedis
}

\author{
Hiroyuki Harada* and Yutaka Tanaka \\ Graduate School of Engineering and Design, Hosei University, Tokyo 102-8160, Japan
}

(Received July 31, 2014; accepted March 23, 2015)

Key words: $\quad$ piezoelectric motor, vibration motor, threshold, human vibration, walking support

The somatic sensation that results from applying a stimulus to the planta pedis is important to maintaining balance and walking around. In this study, we focus on the difference limens of the planta pedis to develop a new device for walking support. Experimental devices for mechanical stimulation by vibration are developed to investigate the two-point threshold level on the planta pedis. Our results clarify that the difference limens depends on the region of the planta pedis.

\section{Introduction}

Recently, devices for walking support and pseudowalking experience have been studied to improve rehabilitation and virtual reality. ${ }^{(1,2)}$ These devices reproduce the sense of walking with a walking motion and convert a real stepping motion into a pseudowalking motion. They also have a large work space and are expensive. Highperformance shoes by making use of a sponge-core-soft rubber actuator have been developed to keep the center of gravity position inside a stipulated area for elderly people. . $^{(3)}$

The body balance necessary for walking is affected by vision, vestibular sensation, and somatic sensation. ${ }^{(4)}$ We can experience pseudowalking only from stepping by displaying a moving image on a monitor. An electric stimulus to the vestibular sensation can control the balance of a human body in the anode direction of the electrode..$^{(5)}$ The somatic sensation perceives physical activity and resistance. We recognize physical stimulation as the somatic sensation through mechanoreceptors, which are plentifully distributed on the planta pedis. ${ }^{(6)}$ The somatic sensation from the planta pedis is the most important sensation in detecting disturbance agitation from the floor. ${ }^{(7)}$ The stimulus from the planta pedis is necessary to maintain body balance and for walking around. However, there are few studies on the impact of somatic sensation on the planta pedis.

*Corresponding author: e-mail: hiroyuki.harada.3a@stu.hosei.ac.jp 
In this study, a concept of a new device for walking support or pseudowalking experience is proposed without the walking motion. Some physical stimulation to the mechanoreceptor is displayed by actuators on the planta pedis in the new device. We feel a continuous stimulation from heel to toe tip when we are walking. To reproduce this continuous physical stimulation, it is necessary to keep the stimulus interval to the planta pedis within a two-point threshold. In this study, we present a prototype of an experimental device to investigate the somatic sensation on the planta pedis for measuring the two-point threshold level.

\section{Stimulus to Planta Pedis and the Two-Point Threshold}

Figure 1 shows the concept of a new device for walking support. The device consists of many vibrating convex pins that stimulate the planta pedis. The surface of the device is not flat. The distance between pins provided by the device is kept within the two-point threshold, which is the distance on the skin separating two pointed stimulators required to experience two rather than one point of stimulation. The two-point threshold represents the minimum interval between the points that can be identified as separate stimulations. Therefore, it is possible to apply stimulus continuously even when two points kept within the two-point threshold stimulate the planta pedis. To clarify the relationship between the stimulus to the planta pedis and the somatic sensation, we investigate the trend of perception of the somatic sensation using the device.

There are two types of stimulus that the plantar perceives from the device. One type of stimulus is applying a constant force continuously. This is called stimulation by displacement. The other type is applying the force periodically. This is called stimulation by vibration. In previous studies, the two-point threshold level on the planta pedis with stimulation by displacement was measured. ${ }^{(8)}$ However, stimulation by vibration has not been sufficiently studied. Therefore, we investigate the two-point threshold level on the planta pedis using two types of stimulus to design the device for walking support. Moreover, we evaluate which type of stimulation is more suitable for stimulating the planta pedis.

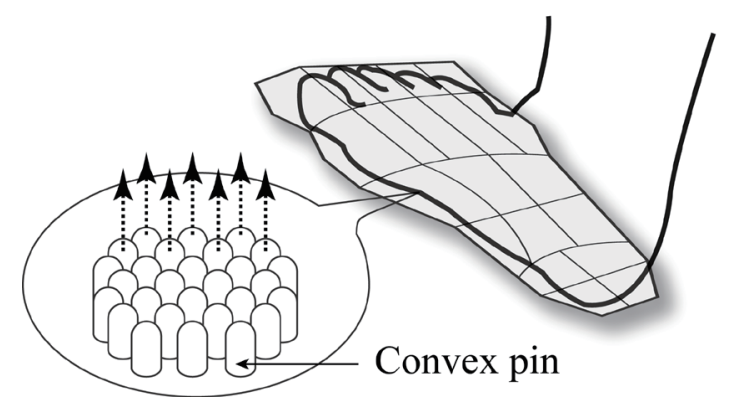

Fig. 1. Concept of the new device for walking support. 


\section{Experiment Using Eccentric Rotating Mass Vibration Motor}

\subsection{Experimental device}

We developed an experimental device that uses an eccentric rotating mass (ERM) vibration motor to perform the experiment on the two-point threshold level with stimulation by displacement and vibration. Figure 2 shows the experimental device using the ERM vibration motor. The pin of the experimental device for stimulating the planta pedis is bonded to the ERM vibration motor. The device can apply stimulation by displacement when it is stopped and by vibration when the ERM motor is running. By moving a part of the device, the distance between two pin points is adjusted in the range of $6.0-80.0 \mathrm{~mm}$.

\subsection{Measurement method}

We measured the two-point threshold level on the planta pedis for five male students in their 20s using the method of limits. We conducted the experiment after each subject was accustomed to the stimulus. The subject was seated on a chair, and his vision and auditory senses were blocked. We pressed the subject's planta pedis with the tips of the pins of the device and measured the two-point threshold level. The subject knew that the stimulus was two points. Figure 3 shows the measurement region of the planta pedis. On the basis of previous studies, stimulus was applied to the ranges of A, B, C, $\mathrm{D}$, and $\mathrm{E}$ in Fig. 3. ${ }^{(3,8)}$ Point $\mathrm{b}^{\prime}$ is the junction of the first metatarsal and the proximal phalanx, and point $\mathrm{c}^{\prime}$ is the junction of the fifth metatarsal and the proximal phalanx. Point $\mathrm{m}$ is determined as the center between points $\mathrm{b}^{\prime}$ and $\mathrm{c}^{\prime}$. Point $\mathrm{e}^{\prime}$ is the center of the heel. We presented stimulation by displacement and vibration and measured the two-

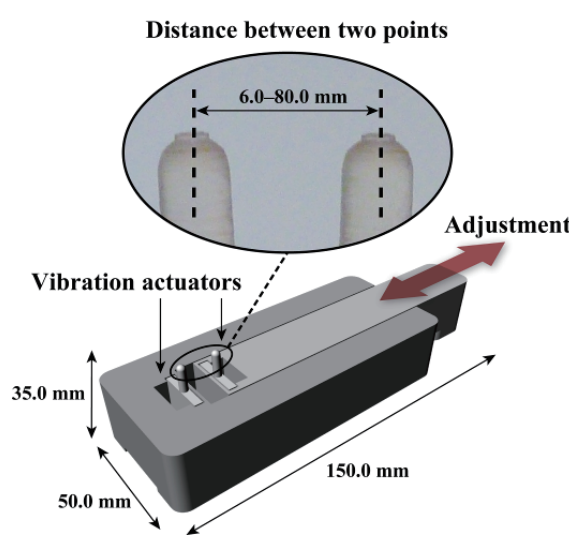

(a)

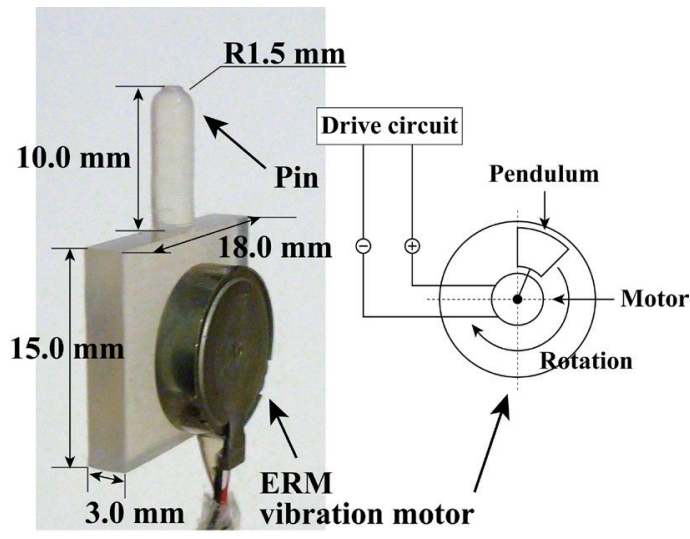

(b)

Fig. 2. (Color online) Experimental device using the ERM vibration motor. (a) Appearance. (b) Vibration actuator. 


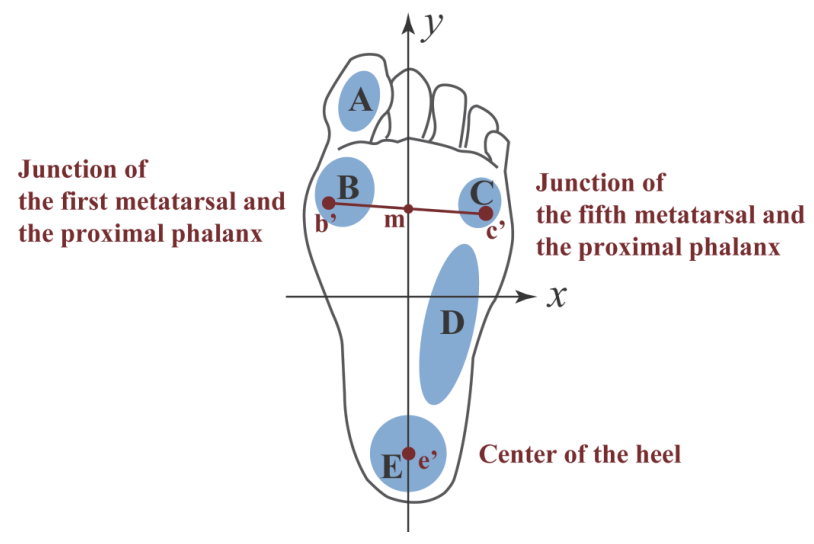

Fig. 3. (Color online) Measurement region of the planta pedis.

point threshold level in the $y$ - and $x$-axis directions (Fig. 3), because current research into stimulation by displacement indicated that the value of the two-point threshold level depends on the direction. ${ }^{(9)}$ The stimulation time was set to $1.5 \mathrm{~s}$. We judged the threshold by three choices: "I feel the two points", "I do not know", and "I feel one point”.

\subsection{Results and discussion}

Figure 4 shows the average threshold of both types of stimulation. The vertical axis shows the threshold level, and the horizontal axis shows the planta pedis region from Fig. 3. In the experiment using the ERM vibration motor, the threshold of the planta pedis for both types of stimulation depended on the region of the planta pedis.

Figure 5 shows the average threshold of both types of stimulation depending on direction. The vertical axis shows threshold level, and the horizontal axis shows the region of the planta pedis from Fig. 3. Green represents the $y$-axis direction, and red represents the $x$-axis direction. The line extending the vertical represents the standard deviation. Most of the thresholds in the $x$-axis direction were lower than those in the $y$-axis direction.

However, there is a positive correlation between the force and the frequency when using the ERM vibration motor because the motor generates force by rotating the pendulum. Therefore, it is difficult to investigate the two-point threshold level by fixing one parameter and generating vibration only in the vertical direction. Moreover, there is a possibility of affecting the values of the two-point threshold level because of inadvertently stimulating the planta pedis in the vertical direction. Accordingly, to clarify the exact relationship between stimulation by vibration and the two-point threshold level, we develop a new prototype of the experimental device and evaluate the relationship. 


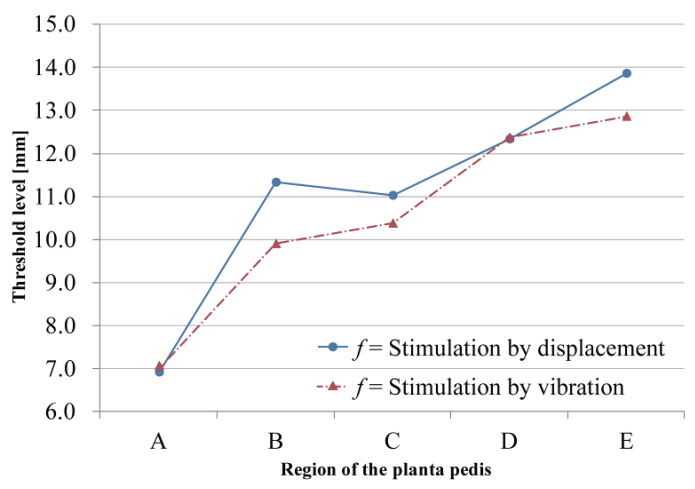

Fig. 4. (Color online) Average threshold of both types of stimulation.

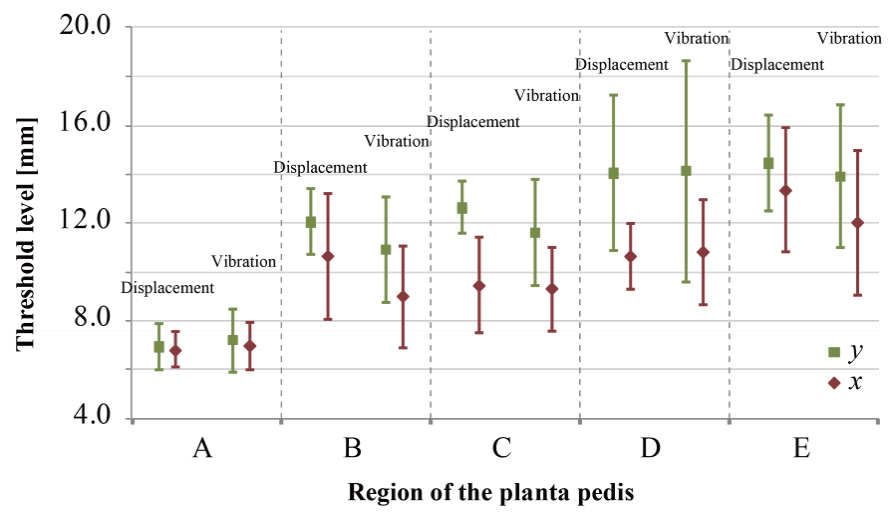

Fig. 5. (Color online) Average threshold of both types of stimulation depending on direction.

\section{Experiment Using Diaphragm-Type Piezoelectric Actuator}

\subsection{Experimental device}

A new experimental device using a diaphragm-type piezoelectric actuator is developed to investigate the two-point threshold level on the planta pedis. Figure 6 shows the experimental device using a diaphragm-type piezoelectric actuator. The ERM vibration motor used in the previous experiments did not keep one parameter fixed. In contrast, it is possible to investigate the two-point threshold level with the diaphragmtype piezoelectric actuator by fixing one parameter because the sound pressure level of the actuator is proportional to the voltage. The pin to stimulate the planta pedis is in contact with the diaphragm-type piezoelectric actuator. The tip of the pin presents different frequency stimulations to the planta pedis by controlling the square wave signal of a microcontroller. The diaphragm-type piezoelectric actuator is turned on or off using a hand switch. A part of the device is moved to adjust the distance between two pins in the range of $3.0-50.0 \mathrm{~mm}$. 


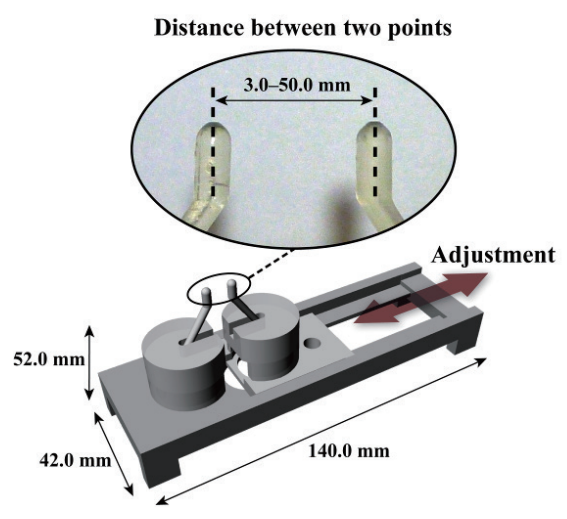

(a)

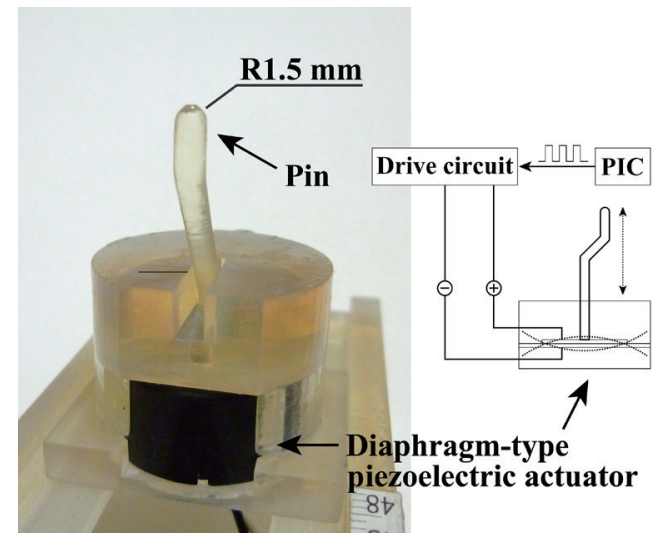

(b)

Fig. 6. (Color online) Experimental device using diaphragm-type piezoelectric actuator. Appearance. (b) Vibration actuator.

\subsection{Measurement method}

We measured the two-point threshold level on the planta pedis of five male students in their 20s using the method of limits, as with the previous experiment. We conducted the experiment after each subject was accustomed to the stimulus. The subject was seated in a chair, and his visual and auditory senses were blocked. We pressed the subject's planta pedis with the tips of the pins of the device and measured the two-point threshold level. The subject knew that the stimulus was two points. Stimulus was applied to the ranges of A, B, C, D, and E from Fig. 3. We presented stimulation of frequency $f=0,100,200$, and $500 \mathrm{~Hz}$, and measured the two-point threshold level in the $y$ - and $x$-axis directions. The stimulation time was set to $1.5 \mathrm{~s}$. We judged the threshold by three choices: "I feel the two points", "I do not know", and "I feel one point".

\subsection{Results and discussion}

Figure 7 shows the average threshold of each frequency stimulus. The vertical axis shows the threshold level, and the horizontal axis shows the region of the planta pedis. The threshold in most of the frequency stimulations depended on the planta pedis region. This result of the two-point threshold is similar to the measurement of the two-point threshold using stimulation by displacement. ${ }^{(8)}$

Figure 8 shows the average threshold of each frequency stimulus depending on direction. The vertical axis shows the threshold level, and the horizontal axis shows the region of the planta pedis. Green represents the $y$-axis direction, and red represents the $x$-axis direction. The line extending the vertical represents the standard deviation. Most of the thresholds in the $x$-axis direction were lower than those in the $y$-axis direction. Therefore, the distance between the convex pins of the new device for walking support needs to be based on the $x$-axis direction, because the $x$-axis direction is easy to perceive than the $y$-axis direction, even when using different frequency stimulations. This result 


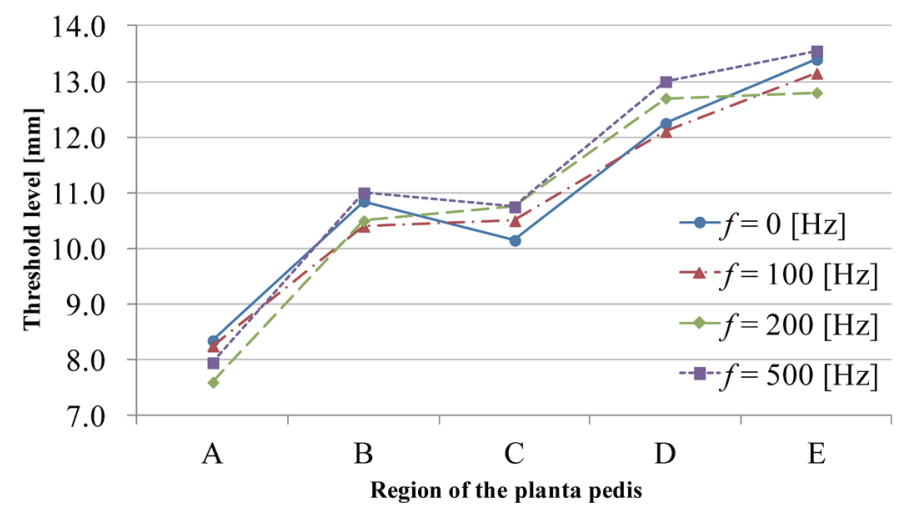

Fig. 7. (Color online) Average threshold of each frequency stimulus.

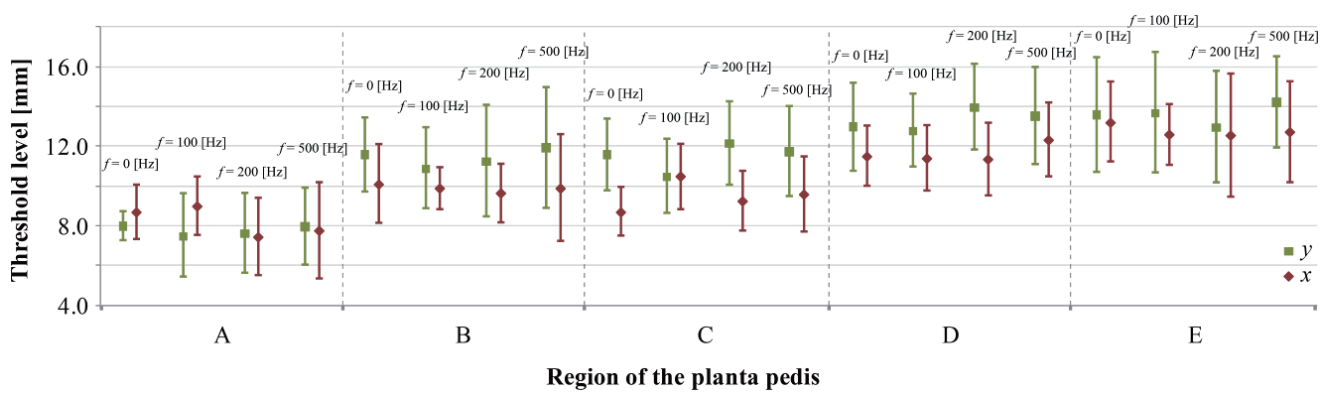

Fig. 8. (Color online) Average threshold of each frequency stimulus depending on direction.

is similar to the existing measurement of the two-point threshold using stimulation by displacement. ${ }^{(8)}$

\section{Conclusions}

In this study, we investigated the two-point threshold level on the planta pedis with frequency stimulations using an ERM vibration motor and a diaphragm-type piezoelectric actuator. Our results clarify that the difference limens depends on the direction and region of the planta pedis. Even when using different frequency stimulations, there is no significant difference between stimulation by displacement and that by vibration. Therefore, stimulation by vibration can be treated in the same manner as that by displacement when considering the two-point threshold.

Future work with the planta pedis stimulation actuator will focus on the hardware development of a new device for walking support, such as the one shown in Fig. 1, and further evaluation of its psychophysical characteristics. 


\section{References}

1 H. Iwata and Y. Yoshida: Presence 8 (1999) 587.

2 R. R. Christensen, J. M. Hollerbach, Y. Xu and S. G. Meek: Presence 9 (2000) 1.

3 Y. Hayakawa, Y. Taguchi and Y. Tooyama: Proc. 8th JFPS International Symposium on Fluid Power (2011) p. 51.

4 O. Blanke: Nat. Rev. Neurosci. 13 (2012) 556.

5 H. Ando, J. Watanabe, M. Sugimoto and T. Maeda: J. Inf. Process. Soc. Jpn. 48 (2007) 1326 (in Japanese).

6 S. Miyajima, T. Matsuda, A. Takanashi, K. Shiota, Y. Nogita, K. Kawada, M. Katou and K. Kurosawa: Rigakuryoho kagaku 27 (2012) 161 (in Japanese).

7 V. Diez, M. Trippel and G.A. Horstmann: Adaptability of Human Gait (Elsevier, North Holland,1991).

8 H. Asai, K. Fujiwara, I. Nara and K. Tachino: Memoirs of Kanazawa University School of Paramedicine 14 (1990) 61 (in Japanese).

9 H. Asai, I. Nara, K. Tachino, K. Fujiwara, H. Togawa and T. Yamashina: J. Phys. Ther. Sci. 2 (1990) 17. 